\title{
Reliability of Internet of Things: smart objects and services
}

\author{
Giacomo Tanganelli ${ }^{1} \cdot$ Marilia Curado $^{2}$
}

Published online: 9 March 2019

(c) Springer Nature Switzerland AG 2019

The special issue is focused on "Reliability of Internet of Things: Smart Objects and Services" and it is composed by four papers. The first two papers are focused on data collection and propagation in smart environments, one is focused on the design of an identification system in combined fog/ cloud architectures, whereas the last one regards the implementation of DC-Net on constrained devices.

Specifically, "Flooding Disaster Resilience Information Framework for Smart and Connected Communities" by Sathish A. P. Kumar, Shaowu Bao, Vivek Singh and Jason Hallstrom, explores methods to fuse social sensor data, physical sensor data, and open demographic data to better react to flooding disasters. Different data representations, coming from different data sources, are unified in a common Space, Time, Theme (STT) format and then aggregated to be further processed, therefore allowing the geo-models to generate more accurate flood predictions. Starting from this point, different research challenges are described that, once addressed, will help to predict hazardous weather events affecting coastal communities. "Smart Application-aware IoT Data Collection" by Vasilios A. Siris, Nikos Fotiou, Alexandros Mertzianis and George C. Polyzos, presents procedures for collection of Internet of Things (IoT) data, from heterogeneous sources, to fulfill different user requirements, namely: data accuracy, data timeliness, energy efficiency and privacy protection. An additive increase and multiplicative decrease (AIMD) adaptive collection is exploited and, when privacy is a concern, noise is added to sensors' measurements by exploiting differential privacy techniques. The evaluation is performed on three different testbeds

Giacomo Tanganelli

giacomo.tanganelli@iet.unipi.it

Marilia Curado

marilia@dei.uc.pt

1 Dip. Ingegneria dell'Informazione, University of Pisa, Pisa, Italy

2 Department of Informatics Engineering, Centre for Informatics and Systems, University of Coimbra, Coimbra, Portugal to collect temperature, humidity and ozone, by means of the FIESTA platform. "Resource Identification in Fog-toCloud Systems: Toward an Identity Management Strategy" by Alejandro Gómez-Cárdenas, Xavi Masip-Bruin, Eva Marín-Tordera and Sarang Kahvazadeh, is focused on the definition of an identify management system (IDMS) that can be exploited to uniquely identify resources in combined fog-to-cloud architectures. The proposed approach associates with each resource a unique identifier, decoupled from the IP address, that remains unchanged even in case of node migration. Performance evaluation is carried out on real hardware (Arduino and Raspberry) to validate the proposed solution. "What it takes to boost Internet-of-Things Privacy beyond encryption with unobservable communication-a survey and lessons learned from a first implementation of DC-net" by Ralf C. Staudemeyer, Henrich C. Pöhls, and Marcin Wójcik, presents an implementation of DC-Net on top of real sensor nodes (Re-Mote). By exploiting DC-Net combined with Mixing, the paper presents a valid prototype that can be exploited to achieve a high level of confidentiality on constrained devices. Furthermore, the paper provides a good survey of available technologies for private communications that can be applied to IoT systems.

The selected papers provide, in our opinion, a good overview of research challenges in the IoT field with particular regards to emerging issues raised, when multiple heterogeneous and distributed IoT systems are combined together.

The guest editors would like to thank the reviewers for the careful analysis of the submissions and the authors for their hard work and contributions.

Publisher's Note Springer Nature remains neutral with regard to jurisdictional claims in published maps and institutional affiliations. 